\section{IN THE NEWS}

Third time lucky?

The future of brain research in the United Kingdom is hanging in the balance, as Cambridge University launches a third appeal for permission to build a primate research facility on the outskirts of Cambridge (BBC News, 25 November 2002). Previous planning applications were rejected because local residents feared that, like the nearby Huntingdon Life Sciences, the centre would provide a target for animal rights protesters.

This time, the opposition to the scheme is being led by animal rights groups. Ray Greek, a former animal researcher who now disputes the validity of animal experiments, is representing the National Anti-Vivisection Society at the hearing. His view is that "chimp brains and human brains are similar in structure, but that doesn't mean they perform the same functions" (New Scientist 23 November 2002).

However, a spokesperson for Cambridge University argues that "advances in the treatment of Parkinson's disease, asthma and strokes have all been made as a result of research in primates.

Research with primates offers the hope of effective treatments for conditions such as Alzheimer's disease and sight disorders, as well as the development of vaccines for malaria and AIDS" (BBC News). John Strandberg, who is responsible for funding decisions on primate research at the National Institutes of Health, points out that "nonhuman primates are the only mammals that develop the senile plaques and neurofibrillary tangles that are symptomatic of Alzheimer's Disease" (New Scientist).

The president of the British Neuroscience Society, Nancy Rothwell, believes that rejecting the plans will not prevent primate research from taking place, and that "it will be done somewhere else where animal welfare might not be so strictly enforced as it is here" (New Scientist).

Heather Wood

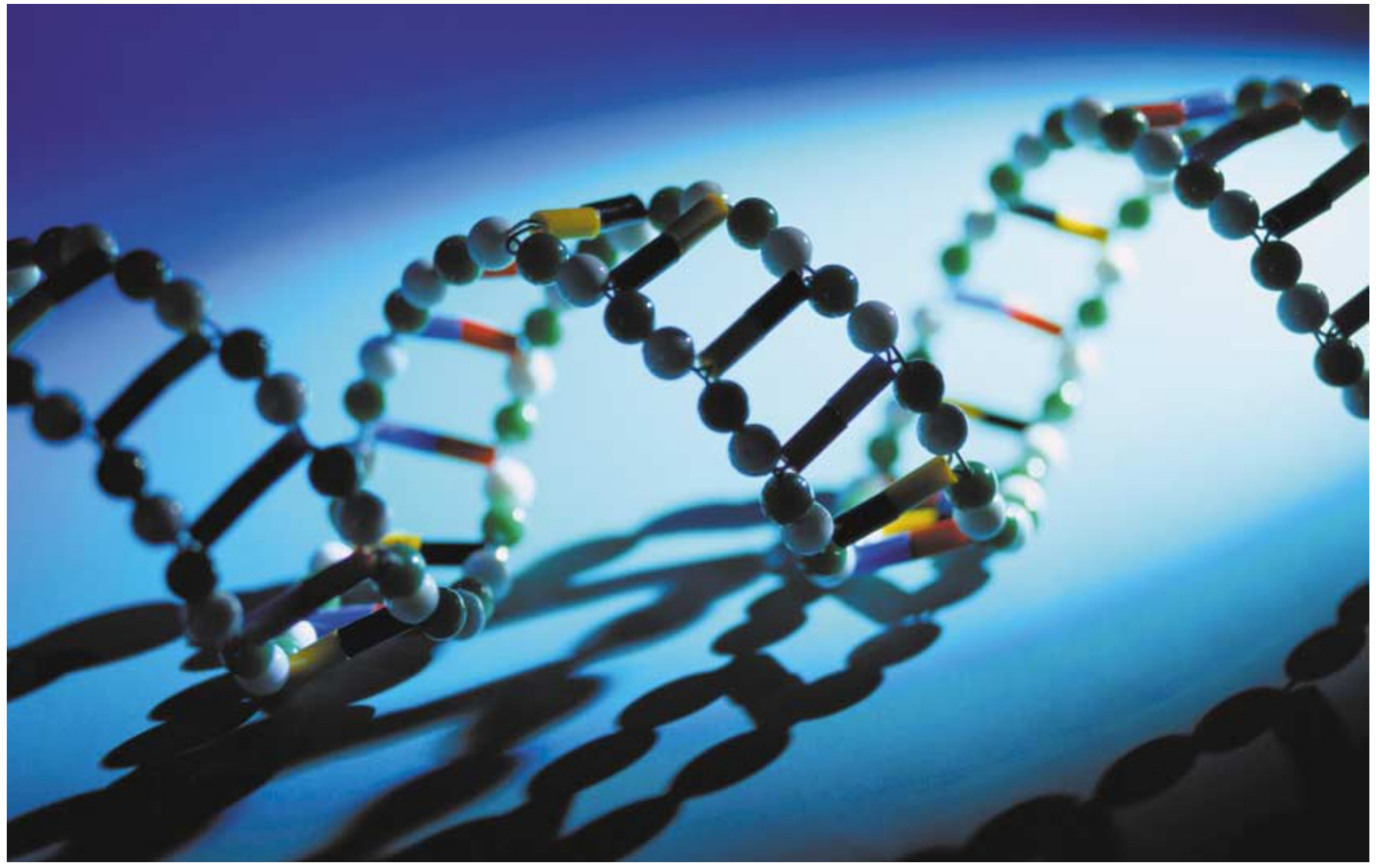

SYNAPTIC PLASTICITY

\title{
The plasticity of chromatin
}

Long-term changes in synaptic efficacy - plastic modifications that might last for hours or even days commonly involve transcription and translation. Some of these activity-dependent changes increase synaptic efficacy, whereas others decrease it. As any given neuron might simultaneously receive potentiating and depressing inputs at different synapses, it is important to find out how the neuron integrates opposing signals. This is particularly interesting if we consider that, owing to the involvement of gene expression, the problem might not be solved locally at the synapse. By studying the nervous system of Aplysia, Guan et al. now provide a possible solution to the dilemma. They report that, when Aplysia neurons receive opposing signals at different synapses, long-term depression predominates over long-term facilitation. Furthermore, they chart the molecular cascade that mediates such an interaction.

Guan et al. cultured Aplysia sensory neurons so that their efferent process bifurcated and contacted two separate postsynaptic motor neurons, making it possible to stimulate the two branches independently. So, if one branch receives repeated doses of serotonin, its synapses show long-term facilitation (LTF). But if the branch receives repeated doses of the neuropeptide FMRFamide, its synapses show long-term depression (LTD). As both plastic processes are specific to the stimulated branch, the authors applied the two stimuli simultaneously to individual branches and asked whether they interacted at all. Indeed, they found that the synapses that received FMRFamide showed LTD, whereas the synapses stimulated with serotonin did not change their efficacy.

LTF involves the phosphorylation of the transcription factor CREB 1 and the subsequent transcription of genes such as CEBP. Guan et al. took this pathway as their starting point and explored whether the interaction between LTF and LTD occurred at this level. Using specific antibodies, they first showed that LTD involved CREB2 but not CREB1, and that CREB2 blocked the induction of CEBP expression in response to serotonin. By performing chromatin immunoprecipitation assays, Guan et al. went on to show that, in response to serotonin, CREB1 recruits the histone acetylase $\mathrm{CBP}$ to the $C E B P$ promoter, leading to the acetylation of histones and the expression of CEBP. By contrast, CREB2 displaced CREB1 from the CEBP promoter, and recruited the histone deacetylase HDAC5. The net effect was a reduction in the expression of CEBP that could account for the overriding effect of LTD over LTF. In fact, Guan et al. came full circle by showing that blocking histone deacetylation prevented the predominating effect of LTD, as the simultaneous application of serotonin and FMRFamide in the presence of deacetylation blockers was accompanied by the expression of LTF in both branches.

Histone acetylation and deacetylation are wellknown mechanisms for the regulation of gene expression, and this paper highlights their involvement in the long-term modification of synaptic function. In addition, the data of Guan et al. give us a glimpse of the way in which neurons can integrate opposing signals at the nuclear level. It will now be important to look for additional effects of LTD-inducing stimuli on chromatin, as it is unlikely that LTD expression involves only the inhibition of transcription and not the expression of specific genes.

Juan Carlos López

(0) References and links

ORIGINAL RESEARCH PAPER Guan, Z. et al. Integration of long-termmemory-related synaptic plasticity involves bidirectional regulation of gene expression and chromatin structure. Cell 111, 483-493 (2002) FURTHER READING Martin, K. C. \& Kosik, K. S. Synaptic tagging who's it? Nature Rev. Neurosci. 3, 813-820 (2002) 